\title{
Ganzheitlicher Ansatz darf nicht verloren gehen
}

Taj H. Zaman

Dr. med. Facharzt FMH für Psychiatrie und Psychotherapie

\author{
Um die Komplexität etwas zu reduzieren: Die Heilkunst ist heute biopsychosozial. \\ Das gilt umso mehr für die psychischen Störungen. Das Studium der klinischen \\ Psychologie ist nicht biopsychosozial. Die dortigen biologischen Inhalte sind illus- \\ trativer Natur. Nicht nur fehlen die Disziplinen, die zur Befähigung der Medika- \\ mentenverschreibung notwendig sind, es fehlen insbesondere die Kenntnisse, die \\ zur Befähigung der psychischen und somatischen, aber eben auch der somatischen \\ und psychischen Differentialdiagnose notwendig sind.
}

Eine biomedizinische Beurteilung ist nicht nur am Anfang der Behandlung, sondern auch in deren Verlauf von eminenter Bedeutung: Es braucht heute nicht nur initial eine biopsychosoziale Untersuchung zum Aus-

\section{Résumé}

La coopération clinique entre médecins et psychologues dans les soins ambulatoires en cabinet - l'importance d'une approche globale

Guérir est une activité biopsychosociale. Les programmes des facultés de médecine sont proposés dans ce sens. L'étude de la psychologie clinique repose, elle, sur une autre tradition sociétale/académique, plus axée sur la compréhension théorique que sur l'atténuation de la souffrance. Dans ce contexte, non seulement la psychologie développe des concepts somatiques trop rudimentaires, mais surtout elle manque d'outils pour comprendre les interactions entre les différents domaines de la santé.

L'activité médicale passe par la relation thérapeutique, notamment dans les troubles psychiques. Cette relation est unique et dépend fortement des personnes. Si elle doit être partagée, cela exige une intense coopération entre les thérapeutes des domaines médicaux et psychologiques.

Ce délicat partage est souvent évité. Les médecins délégants, notamment, négligent leur mission et ne voient jamais les patients délégués. Cela aboutit à des erreurs de traitement et entraîne des erreurs médicales.

Si les conditions professionnelles, sociologiques, sociétales et politiques exigent la répartition du traitement entre plusieurs praticiens, mais que dans la réalité ce partage est en grande partie mal géré, les organes étatiques responsables doivent mettre en place une structure qui garantisse le critère de qualité d'une étroite coopération entre les professions. Autrement l'erreur médicale sera institutionnalisée.

En cas d'excédent de demandes, l'augmentation des prestataires ne permettra pas de faire des économies. schluss von organischen Ursachen und Einflüssen auf die psychische Symptomatik, auch im Behandlungsverlauf wirken ständig biologische Faktoren auf den psychischen Zustand ein, die der geisteswissenschaftlich-psychologisch ausgebildete Psychotherapeut nicht wird erkennen können:

Liegt eine neu aufgetretene Erkrankung vor wie z.B. eine subklinische Infektion mit einzig psychischen Auswirkungen, eine neue somatische Medikation mit möglichem Einfluss auf die psychischen Funktionen, eine Fluktuation im Verlauf einer chronischen somatischen Erkrankung, gar einer solchen mit schwieriger $\mathrm{Zu}$ weisung der Symptomatik zum somatischen oder psychischen Bereich - in all diesen Situationen braucht es nicht nur integrative biopsychosoziale Kenntnisse zur gesundheitlichen Beurteilung, sondern auch viel Erfahrung. Und doch bleibt dies mit einem grossen Unsicherheitsfaktor behaftet. Da ist die Medizin trotz aller technisch-biologischen und technisch-psychologischen (Psychotherapie-«Manual») Fortschritte reine Kunst. Ein weiteres Problem bilden schwer beeinträchtigte $\mathrm{Pa}$ tienten, die sich ohne wochen- bis monatelange Beziehungsarbeit (Motivation zum Eingehen einer Arzt/Therapeut-Patient-Beziehung, welche das Medium der Behandlung psychischer Störungen ist) nicht auf eine therapeutische Beziehung einlassen können und damit oft eine indizierte Medikation ablehnen. Findet nun diese intensive Beziehungsarbeit bei einem psychologischen Psychotherapeuten statt, so ist es häufig nicht mehr möglich, diese Therapie mit indizierter Medikation zu ergänzen, welche Medikation hingegen bei Durchführung der erwähnten Beziehungsarbeit durch 
einen Facharzt für Psychotherapie (und Psychiatrie) eine logische Ergänzung der Behandlung sein wird.

\section{Zwei grundlegend verschiedene Berufsfelder}

Trotz all dieser Schwierigkeiten für Interventionen im therapeutischen Medium «Beziehung» delegiere ich seit vielen Jahren die ärztliche Psychotherapie teils an Fachpsychologen, teils an Psychologen in Weiterbildung (letztere findet nicht unter meiner Verantwortung statt). Dies ist eine hervorragende Gelegenheit, um zu beobachten, was geschieht, wenn die Behandlung auf zwei Behandler aufgeteilt wird, denn trennen lassen sich die psychosozialen und die biologischen Interventionen nie vollständig - ganz im Sinne biopsychosozialer Logik. In diesem «Berufsfeldlabor» fehlt leider die Möglichkeit der Forschung nach anerkannter soziologischer Methodik.

Hier geht es auch ums Verständnis des multiprofessionellen Arbeitens. Dazu gehört die Definition einer gemeinsamen Fachsprache, was je nach mitgebrachten Voraussetzungen sehr schwierig sein kann (Kenntnisse der Psychopathologie, Trennung von Untersuchung, Beurteilung und Intervention) trotz formal universitärer Ausbildung in Psychopathologie. Meist gelingt mit der Zeit die Entwicklung einer gemeinsamen Sprache,

Es geht ums Verständnis des multiprofessionellen Arbeitens. Dazu gehört die Definition einer gemeinsamen Fachsprache.

es bleibt aber immer ein Unsicherheitsfaktor, der im Verlauf der Behandlung durch intensives Zusammenarbeiten weitgehend ausgeräumt werden kann.

Erst diese intensive Zusammenarbeit macht die Trennung der «Integrierten psychiatrisch-psychotherapeutischen Behandlung" (Facharztcurriculum) in einen fachpsychologischen und einen fachärztlichen Teil möglich. Wöchentlich und bei speziellen Situationen häufiger werden Patienten besprochen und die weitere Behandlung geplant. Dabei bleibt die Psychotherapie immer delegiert, gegebenenfalls muss der psychologische Psychotherapeut in der eigenen Intervision oder Supervision schwierige Situationen klären. Der allgemeine Teil der Psychotherapie hingegen wird gegenseitig abgesprochen. Diese allgemeine Psychotherapie umfasst vorwiegend die strukturellen Voraussetzungen inkl. der Behandlung durch zwei Fachpersonen, dann die Diagnostik (welche in unserem Fach immer hinterfragt und teils revidiert werden muss), aber auch grundlegende Aspekte des Settings und der Gestaltung der therapeutischen Beziehung. Über die Pharmakotherapie ist der Psychologe immer informiert (wie auch andere, z.B. der Apotheker, der das Rezept ausführt).
Manchmal werden zur Klärung der psychopharmakologischen Indikation die psychopathologischen Erkenntnisse und psychotherapeutischen Interventionen aus der delegierten Psychotherapie herangezogen und auf diese abgestimmt. Einerseits ist die Verordnung eines Medikamentes nie eine rein technische Angelegenheit; der Verordner ist kein Medikamentenspender Anderseits beherbergt jede psychopharmakologische Intervention auch Anteile einer psychosozialen Intervention, die psychotherapeutisch ausgerichtet ist bzw. sein muss (z.B. gezielter Einsatz des Placeboeffekts). Hier erneut ist der Austausch zwischen den beiden Fachleuten von zentraler Bedeutung [1].

\section{Unbefriedigende Realität}

Die Delegationen von Psychotherapie werden aber real auch anders gehandhabt: Der delegierende Arzt sieht den Patienten nie, delegiert also die ganze Behandlung dem Psychologen. Oder der Arzt sieht den Patienten einmal, verordnet ein Medikament und erneuert das Rezept jährlich ohne eine weitere Konsultation. Oder der Patient konsultiert den Arzt erst, wenn der Psychologe ihn dazu drängt. Wie der Psychologe die Indikation für eine Medikation stellt, bleibt unklar. Häufig ist dies der Fall, wenn die alleinige Psychotherapie nicht die erwünschten Resultate ergibt. Wir wissen als Ärzte natürlich, dass dies keine gute Indikation sein kann. Nicht selten bleibt der Patient über lange Zeit unterdosiert: in der Behandlung psychischer Störungen einer der häufigsten Kunstfehler. Denn eine Unterdosierung bringt meist Schaden. Dies können unmittelbare metabolische Auswirkungen sein, aber auch die Medikamentenresistenz kann so gefördert werden: Die Teilwirksamkeit wird zu einer Restwirksamkeit und verschwindet.

Diese Fehler der Behandlung können durch eine intensive, multiprofessionelle Zusammenarbeit Arzt-Psychologe vermieden werden. Nur dieses multiprofessionelle Vorgehen kann die geforderte Ergebnisqualität garantieren. Ich gehe davon aus, dass dies die Absicht des Staates war, als er die delegierte Psychotherapie einführte: ärztlich angestellt und in der ärztlichen Praxis arbeitend. Es ging kaum um den Schutz des ärztlichen Berufsstandes. Leider wurden in der Praxis diese Bedingungen schnell umgangen. Die Anstellung wird als Mandatserteilung verstanden, oder die Praxis des Psychologen wird als ärztliche Filialpraxis deklariert. Nicht nur in diesen Fällen ist die Behandlung zur Gänze delegiert: Der Arzt weiss nichts von der Behandlung, und erhält auch keinen Bericht vom behandelnden Psychologen. Und dies, obwohl die Schweizerische Gesellschaft für Psychiatrie und Psychotherapie (SGPP) schon vor bald zehn Jahren die Delegation der ganzen Behandlung 
ausdrücklich ausgeschlossen hat [2]: Wie kann ein Arzt die Verantwortung für die Behandlung eines Patienten übernehmen, den er nicht gesehen hat? Auch dem Rechtsdienst der FMH ist sich die Sache dubios.

Ein delegierender Arzt (auch wenn er den «Fähigkeitsausweis delegierte Psychotherapie» besitzt) addiert zu einem psychologischen Psychotherapeuten ergibt eben noch lange keine integrative biopsychosoziale Behandlung - die wäre nur durch eine enge multiprofessionelle Einstellung möglich.

Gerade eine solche biopsychosoziale Behandlung ist der Hausarztmedizin wichtig. Es wird dort, und auch in anderen Spezialitäten, eine mangelnde Berücksichtigung der psychosozialen Determinanten der Gesundheit geortet. Deswegen wird nachvollziehbar, wenn der mfe («Haus- und Kinderärzte Schweiz») und andere ärztliche Vereinigungen die psychologischen Aspekte im Gesundheitswesen fördern wollen. Leider genügt es dazu nicht, die Psychologen in ihrem Bestreben, am Gesundheitskuchen zu partizipieren, zu unterstützen. Die Integration des Psychosozialen in die Hausarzt- (und andere) Medizin kann nicht gelingen, indem die Verantwortung für die psychosozialen Determinanten der Gesundheit an eine weitere, nicht ärztliche Profession übergeben wird. Im Gegenteil: Die Lockerung der gesetzlichen Bindung Arzt-Psychologe wird selbstverständlich zu einer weiteren Zementierung des biopsychischen Dualismus führen und die ursprüngliche Idee der Ergänzung wird zum Fortschreiten der Trennung führen. Psychologen sind von ihrer Ausgangsmotivation und von ihrer beruflichen Sozialisation her Spezialisten für die Psyche. Ihre Erfahrung beinhaltet keine intensive, höchstens eine theoretische Beschäftigung mit dem physischen Körper. Und sie spüren dieses Defizit, zumindest im akademischen Bereich, wo zunehmend reduktionistische biologische Konzepte zur Erklärung intrapsychischer oder interaktioneller Phänomene herangezogen werden. So werden mit der Zeit Fachspezialisten für das «Organ Psyche» entstehen mit Teilkenntnissen über die neuronale Funktion bspw., die - nach dem biomedizinischen Modell konzipiert und zu anderen Fachspezialisten addiert - die Illusion der Ganzheitlichkeit zu erwecken versuchen werden.

\section{Wie soll es weitergehen?}

Die Verantwortung der staatlichen Institutionen kann nicht darin bestehen, die Umgehung des bisherigen Systems einer engen Zusammenarbeit PsychologeArzt durch ein Anordnungsmodell zu zementieren. Die Verantwortung der staatlichen Institutionen besteht darin, eine soziopolitische Struktur zu schaffen, die dem Qualitätsbedürfnis und Qualitätskriterium der «engsten multiprofessionellen Zusammenarbeit» entgegenkommt und diese garantiert. Da genügt die Pflicht zu häufigen schriftlichen Berichten nicht. Es muss mit Bezug auf die Behandlungsqualität analysiert werden, wo sich die delegierte Psychotherapie bewährt hat und wie sie verbessert werden kann, jenseits berufspolitischer Forderungen. Der Bundesrat soll die jetzige Situation nutzen, um die Versorgungsforschung voranzutreiben. Die Situation in Deutschland zeigt unerwünschte Wirkungen, die wahrscheinlicher werden, wenn nicht von der Sache (Behandlung von psychischen Störungen) ausgegangen wird. Auch in der Schweiz sind unerwünschte Wirkungen ähnlich wie in Deutschland denkbar, wenn nicht von der Sache (Behandlung von psychischen Störungen) ausgegangen wird.

Die Föderation der Schweizer Psychologinnen und Psychologen (FSP) verlangt eine totale Autonomie und will lediglich eine ärztliche Verordnung akzeptieren, weil eine direkte Behandlung politisch noch nicht durchsetzbar sei [3]. Die ökonomische Verantwortung will sie aber der Ärzteschaft überlassen [4]. santésuisse wird aber die Rückforderung nach den veranlassten, sprich verordneten (psychotherapeutischen) Kosten richten.

\section{Fragliche Argumentation}

Auch die FSP weiss, dass "ambulant vor stationär» nicht automatisch Kosten spart. Das kann dort nicht zutreffen, wo ein Nachfrageüberhang besteht. Und gerade dieser Überhang dient ihm als Rechtfertigung für den Zugang zu der obligatorischen Krankenpflege.

Auch der Vorwurf des «bequemen Mitverdienens der Psychiater» [5] ist fehl am Platz: Im aktuellen Tarif rechnet sich die delegierte Psychotherapie betriebswirtschaftlich niemals: Es können im besten Fall, d.h. bei einer langjährigen Anstellung eines fachlich und in der Zusammenarbeit mit Ärzten erfahrenen Fachpsychologen, die laufenden Kosten gedeckt werden. Abgeschrieben werden müssen jedoch die Investitions- und Anstellungskosten. Aber dieser Vorwurf erstaunt kaum aus dem Munde zweier Psychologen, die die enge $\mathrm{Zu}$ sammenarbeit mit der Ärzten als «absurd» [6] oder im YouTube-Video des Psychologenverbandes FSP als «diskriminierend» und «unwürdig» bezeichnen [7].

Literatur

1 Psychology of Consciousness: Theory, Research, and Practice 2016, Vol. 3, No. 2 .

2 Zitiert in https://www.vertrauensaerzte.ch/expertcom/psyamb. html

3 Homepage der FSP.

4 Yvik Adler, Stephan Wenger, Co-Präsidenten FSP, SÄZ 2019;100(21): 722.

5 Schweiz Ärzteztg. 2019;100(19):646-647.

6 Stephan Wenger, FSP, «Tribune de Genève», 17.11.18.

7 https://www.youtube.com/watch? $=\mathrm{dH}$ mseUOXcO\&feature=youtu.be\&t $=145$ 